\title{
NEOVASCULAR COMPLICATIONS AFTER CENTRAL RETINAL VEIN OCCLUSION
}

\author{
K. EVANS, P. K. WISHART and J. N. McGALLIARD \\ Liverpool
}

\begin{abstract}
SUMMARY
An 8-year retrospective study of patients suffering a central retinal vein occlusion (CRVO) was undertaken to study secondary new vessel formation and whether pathologies known to predispose to CRVO influenced the occurrence of these neovascular complications and their responsiveness to treatment. Seventy-three patients were studied. Ocular neovascularisation had occurred in $60 \%$. More specifically $39 \%$ developed neovascular glaucoma. Panretinal photocoagulation produced regression in only $37 \%$ of those with established neovascularisation and was unsuccessful in preventing neovascularisation in five patients treated prophylactically. Patients with pre-existing primary open angle glaucoma (POAG) were statistically more likely to develop ocular neovascularisation $(p=0.02)$, which was also less responsive to laser therapy $(p=0.02)$. Adequate prior glaucoma therapy did not protect against this enhanced complication rate. It was concluded that POAG is a significant risk factor for developing ocular neovascularisation after CRVO which will be refractory to laser therapy.
\end{abstract}

Central retinal vein occlusions (CRVOs) are often complicated by the development of ocular neovascularisation. Retinal hypoxia alters the balance of vasoproliferative and inhibitory growth factors in the eye, stimulating new vessel growth. ${ }^{1}$ The anterior segment is most commonly affected with new vessel formation of the iris (rubeosis) and iridocorneal angle. Neovascular glaucoma (NVG) is diagnosed when this neovascularisation causes occlusion of the drainage angle resulting in a severe rise in intraocular pressure and an inflamed, painful eye. Occasionally new vessel formation develops at the optic nerve head or more rarely the retina. ${ }^{2}$

Recognising these complications and identifying those at risk is a major concern for the ophthalmologist managing CRVO patients. Success at preventing or reversing new vessel formation has been reported ${ }^{3,4}$ if patients are treated early with panretinal photocoagulation. Not all

Correspondence to: P. K. Wishart, FRCS, FCOphth, St Paul's Eye (Glaucoma) Unit, Royal Liverpool University Hospital, Prescot Street, Liverpool L7 8XP, UK.
CRVOs are complicated by neovascularisation and since retinal laser treatment is not without adverse effects, great efforts are usually expended on early identification of those who would benefit. However, this can be difficult, ${ }^{5,6}$ and indeed the effectiveness of panretinal photocoagulation has recently been questioned. ' With these thoughts in mind we undertook a study to document the occurrence and outcome of neovascular complications in CRVO patients and their responsiveness to treatment, particularly panretinal photocoagulation. Also, to expedite future identification of those who will develop ocular neovascularisation, we proposed to assess whether the presence of any of the pathologies thought to predispose to CRVO, such as diabetes, hypertension or primary open angle glaucoma, influenced the likelihood of neovascular complications and their responsiveness to treatment.

\section{METHODS}

A retrospective study was undertaken of patients under the care of two consultant ophthalmologists who had presented with a CRVO between 1982 and 1990 (8 years). The list of patients was compiled from the Ophthalmic Casualty and Laser Treatment Registers, the Fluorescein Angiography Database and by the authors screening outpatient records. Only patients with at least 2 years of documented follow-up were included. The diagnosis of CRVO had been based on a typical history of sudden loss of vision and clinical examination findings of widespread retinal haemorrhages in all four quadrants, venous engorgement and signs of retinal oedema or ischaemia. The diagnosis in most cases had been confirmed by fluorescein angiography.

The following information on patients was collated from the case notes:

1. Age and sex.

2. Past general medical history and the results of investigations performed at presentation to identify pathologies thought to be associated with CRVO.

3. Past ophthalmic history and details of ocular examination at the time of presentation with a CRVO and at 
subsequent visits. NVG had been diagnosed when anterior segment neovascularisation was associated with an intraocular pressure (IOP) above $40 \mathrm{mmHg}$ causing a red, painful eye.

4. Methodology of ocular treatment regimens used and their outcomes.

The diagnosis of pre-existing primary open angle glaucoma (POAG) can be difficult in CRVO patients. A drop in IOP following a CRVO is well-documented; ${ }^{8}$ therefore, a 'normal' IOP in the affected eye would not exclude POAG. The cup/disc ratio and the appearance of the neuroretinal rim in the affected eye are not reliable indicators since optic nerve head morphology is often masked by peripapillary haemorrhage and disc oedema. Field analysis in the affected eye would also be difficult to interpret. Because POAG is commonly a bilateral condition ${ }^{9}$ we have inferred that its presence in the fellow eye is strongly suggestive of pre-existing POAG in the affected eye. Therefore a diagnosis of pre-existing POAG was made either if there was a clear past ophthalmic history of this, or if at presentation there was raised IOP, disc cupping and visual field loss in the fellow eye which was not due to other ocular disease and which subsequently required glaucoma treatment. POAG patients were subclassified according to whether IOP was 'controlled' or 'uncontrolled'. Uncontrolled IOP was defined as IOP measurements above $21 \mathrm{mmHg}$ on a number of occasions in the year preceding CRVO. Those whose pre-existing POAG was not diagnosed until presentation with a CRVO were included in the uncontrolled sub-group.

An HGM model 5 argon laser (Medical Laser Systems) on blue-green setting had been used for panretinal photocoagulation with either a Mainster or Goldmann 3 mirror contact lens. Patients had been offered panretinal photocoagulation if there was evidence of significant retinal ischaemia on fluorescein angiography, or if new vessels were found on clinical examination or angiography. ${ }^{2}$ The underlying philosophy of treatment had been to use as much power as necessary to obtain an adequate burn, treating as much retina as possible outside the macular region.

Statistical analysis was carried out using Statistical Analysis System (SAS), Release 6.03 (SAS Institute, Cary, NC) on an Olivetti PCS286 personal computer. Con- tingency tables, chi-squared statistics and Fisher's exact test (two-tailed) were carried out using the FREQ procedure. The effect of age on outcomes was assessed using TTEST. Logistic regression, ${ }^{10}$ to examine the influence of confounding variables in combination, was conducted using the LOGIST procedure.

\section{RESULTS}

Ninety-three cases of CRVO were identified for the study period. A full 2 years of documented follow-up was not available for 20 cases and these were excluded from further analysis. The remaining 73 patients comprised the final study population. The mean age was 71.2 years (range 30-87 years). Forty-two (58\%) were female and 31 (42\%) male. In 41 cases one pathology predisposing to CRVO was identified, in 9 individuals two predisposing pathologies were identified and in 6 patients three. These included cases of POAG, ocular hypertension, diabetes mellitus, systemic hypertension, peripheral vascular or cardiac disease and hyperlipidaemia (Table I).

Forty-four patients $(60 \%)$ had developed ocular neovascularisation diagnosed on the basis of clinical examination and fluorescein angiography. Seven patients had developed optic nerve head or retinal new vessels and 8 had developed rubeosis irides. NVG had been diagnosed in 29 (39\%). In 12 cases NVG had been diagnosed at presentation.

Table I documents the occurrence of ocular neovascularisation with each predisposing pathology identified. Only the association of POAG with ocular neovascularisation was statistically significant $(p=0.01)$. Fig. 1 illustrates that most cases of neovascularisation in POAG patients had progressed to NVG. No association with patient age was found using Student's $t$-test $(t=-0.9$, $p=0.37$ ).

The diagnosis of pre-existing POAG had been made on the basis of an established past ophthalmic history in 15 cases and was diagnosed at presentation with a CRVO in 17. The POAG sub-group included 4 of the diabetics, 8 of the hypertensives and 6 of those suffering peripheral vascular or cardiac disease who had been identified in the total study population. POAG patients had a mean age of 69.4 years (range 30-84 years). Logistic regression analysis using a main effect model was performed to correct for the confounding influence of patient age and other pathol-

Table I. Association between ocular neovascularisation and response to panretinal photocoagulation with factors predisposing to CRVO

\begin{tabular}{|c|c|c|c|c|c|c|}
\hline & POAG & $\begin{array}{c}\text { Ocular } \\
\text { hypertension }\end{array}$ & $\begin{array}{l}\text { Diabetes } \\
\text { mellitus }\end{array}$ & Hypertension & $\begin{array}{c}\text { Peripheral } \\
\text { vascular disease }\end{array}$ & Hyperlipidaemia \\
\hline No. in study & 32 & 3 & 10 & 20 & 12 & 2 \\
\hline Developed NV (\%) & $25(78)$ & $0(0)$ & $4(40)$ & $9(45)$ & $8(67)$ & $1(50)$ \\
\hline$\chi^{2}(p$ value $)$ & $6.3(0.01)$ & $(0.06)^{\mathrm{a}}$ & $(0.14)^{\mathrm{a}}$ & $1.8(0.17)^{\mathrm{a}}$ & $(0.43)^{\mathrm{a}}$ & $(0.64)^{a}$ \\
\hline Odds ratio & 4.1 & $\infty$ & 2.6 & 0.4 & 1.3 & 1.5 \\
\hline$(95 \% \mathrm{CI})$ & $(1.4-11.7)$ & $(0.65-\infty)$ & $(0.54-13.8)$ & $(0.15-1.2)$ & $(0.32-6.9)$ & $(0.02-123)$ \\
\hline Laser treatment & 18 & 0 & 3 & 6 & 4 & 0 \\
\hline Failure of $\mathrm{NV}$ regression $(\%)$ & $15(84)$ & - & $1(33)$ & $2(33)$ & $4(100)$ & - \\
\hline$\chi^{2}(p$ value $)$ & $4.9(0.02)$ & - & $(0.54)^{\mathrm{a}}$ & $(0.17)^{\mathrm{a}}$ & $(0.27)^{a}$ & - \\
\hline Odds ratio & 7.14 & - & 0.26 & 0.19 & 0 & - \\
\hline$(95 \% \mathrm{CI})$ & $(1.5-34.4)$ & - & $(0.004-5.8)$ & $(0.02-1.7)$ & $(0-2.5)$ & - \\
\hline
\end{tabular}

POAG, primary open angle glaucoma; NV, new vessels; CI, confidence interval; ' $p$ value determined by Fisher's exact test. 


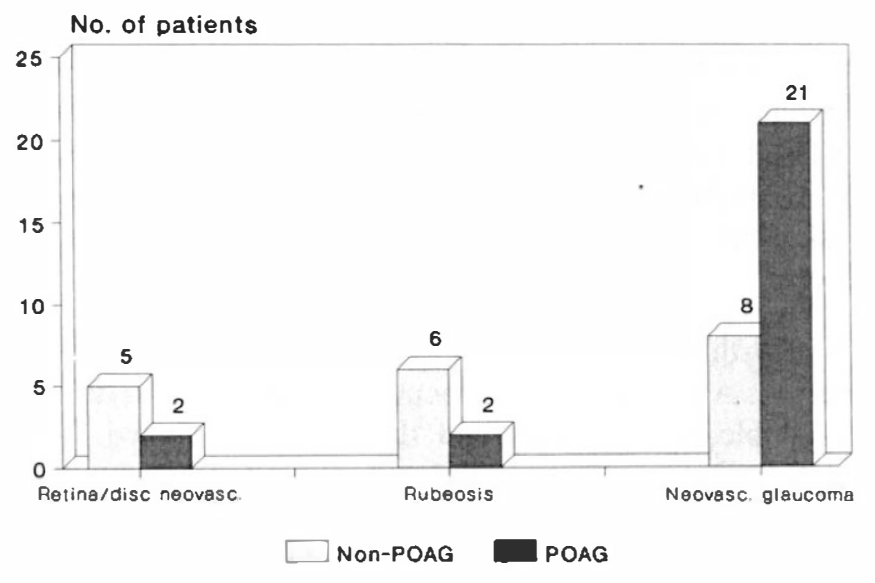

Fig. 1. Neovascularisation amongst those with and without primary open angle glaucoma (POAG).

ogies predisposing to CRVO. The association of POAG with neovascularisation was still statistically significant $(p=0.02$, beta $=1.29$, odds ratio $=3.63 ; 95 \%$ confidence interval $=3.25-8.41)$. The best model predicting neovascularisation contained POAG only (sensitivity $=57 \%$, specificity $=76 \%$, correct prediction in $65 \%$ of cases).

The sub-group of 32 patients with pre-existing POAG was further divided according to whether their IOP was 'controlled' or 'uncontrolled' prior to their CRVO. Thirteen of 18 POAG patients with uncontrolled IOP developed ocular neovascularisation. This compared with 12 of 14 POAG patients with good IOP control who developed ocular neovascularisation. The difference in incidence of neovascularisation between POAG patients with uncontrolled or controlled IOP was not statistically significant (Fisher's exact test, $p=0.4$ ).

Forty patients had undergone panretinal photocoagulation. All 5 who had been treated prophylactically subsequently developed neovascularisation. A beneficial response to laser treatment, i.e. regression of ocular neovascularisation as assessed by clinical examination, was seen in only 13 of the 35 with neovascularisation already present $(37 \%)$. Regression was seen in only 5 of 22 neovascular glaucoma cases (23\%). Four patients with established ocular neovascularisation had not undergone laser No. of patients

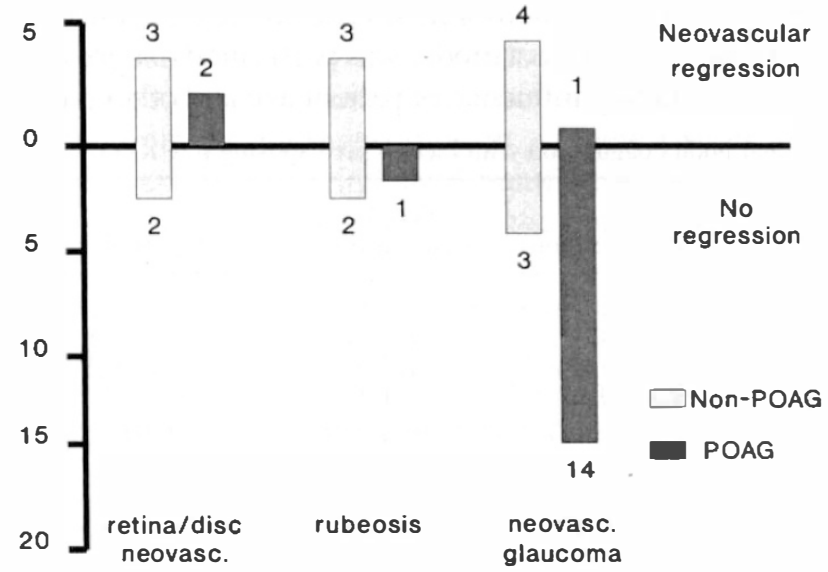

Fig. 2. Response to laser therapy amongst those with and without POAG. treatment. Two had refused treatment and poor fundal view had precluded treatment in the other 2 .

Patient age and each pathology predisposing to CRVO were studied to determine whether they influenced the outcome of laser treatment (Table I). A statistically significant association was found between POAG and laser treatment outcome $(p=0.02)$. An odds ratio of 7.14 (1.5-34.4) indicated that this association was with failure of regression with treatment. Fig. 2 illustrates this poorer response amongst POAG patients. A significant association between a poor response to laser treatment and advancing age was also found $(t=2.8, p=0.01)$. To avoid error from model overfitting, ${ }^{10}$ logistic regression was performed using these two significant variables only. This best model gave a sensitivity of $91 \%$, a specificity of $62 \%$ and a correct prediction of poor response in $80 \%$ of cases. Correcting for the influence of age, the independent influence of POAG on poor treatment outcome was still statistically significant $(p=0.02$, beta $=2.14$, odds ratio $=$ 8.6 ; $95 \%$ confidence interval $=2.0-50.5$ ). Table II documents that the panretinal photocoagulation regimens used in those with and without POAG were comparable.

Thirteen NVG patients had undergone IOP-controlling surgery which included 4 trabeculectomies ( 2 were successful at lowering IOP), 8 cyclocryotherapies ( 2 successful) and 1 single-plate Molteno tube insertion (unsuccessful at lowering IOP). Pre-existing POAG was not more prevalent amongst those whose surgery was unsuccessful ( 1 of 9 ).

Table III documents the final visual acuities at 2 years of those with and without POAG. Most patients (including 17 NVG cases) had a final acuity of 'hand movements' or better. 'No perception of light' had been recorded for 12 patients. The difference in acuities at 2 years between those with and without POAG was not statistically significant (chi-squared $=0.42, p=0.81$ ). Forty-three of 44 patients who had developed ocular neovascularisation had comfortable eyes ( 2 phthisical) at 2 years. Two NVG cases were comfortable at 2 years with just topical medical therapy. One eye had been enucleated for intractable pain.

\section{DISCUSSION}

Great efforts were made to obtain all records of CRVO patients for the study period. Rapid onset of visual loss and/or widespread retinal pathology observed by the general practitioner or optician usually results in referral to a Casualty Department. During the study period it was established practice for all CRVO patients to undergo fluorescein angiography and to treat with panretinal photocoagulation any who had significant retinal ischaemia or established neovascularisation. Therefore by screening records from the Casualty Department, Fluorescein Angiography and Laser Treatment Registers and by reviewing out-patient records the authors doubt that many cases were missed. We conclude that our observations accurately reflect actual clinical experience and practice during the study period.

The incidences found in this study of ocular neovas- 
Table II. Mean values for laser treatment indices for patients with and without POAG

\begin{tabular}{|c|c|c|c|c|c|c|}
\hline & $\begin{array}{l}\text { Days to } \\
\text { treatment }\end{array}$ & $\begin{array}{c}\text { No. of } \\
\text { sessions }\end{array}$ & No. of burns & $\begin{array}{c}\text { Burn } \\
\text { size }(\mu \mathrm{m})\end{array}$ & $\begin{array}{l}\text { Duration } \\
(\mathrm{ms})\end{array}$ & $\begin{array}{l}\text { Power } \\
\text { (W) }\end{array}$ \\
\hline Non-POAG & $\begin{array}{c}55 \\
(10-110)\end{array}$ & $\begin{array}{c}2 \\
(1-5)\end{array}$ & $\begin{array}{c}3185 \\
(1450-3450)\end{array}$ & $\begin{array}{c}200 \\
(200-500)\end{array}$ & $\begin{array}{c}0.1 \\
(0.1-0.2)\end{array}$ & $\begin{array}{c}0.8 \\
(0.4-1.5)\end{array}$ \\
\hline POAG & $\begin{array}{c}58 \\
(2-98)\end{array}$ & $\begin{array}{c}2 \\
(1-3)\end{array}$ & $\begin{array}{c}3310 \\
(1560-3500)\end{array}$ & $\begin{array}{c}200 \\
(200-500)\end{array}$ & $\begin{array}{c}0.1 \\
(0.1-0.2)\end{array}$ & $\begin{array}{c}0.7 \\
(0.3-1.9)\end{array}$ \\
\hline
\end{tabular}

Values in parentheses are ranges.

cularisation in general $(60 \%)$ and of NVG in particular (39\%) following CRVO are comparable with previously published research $\left(66.7 \%\right.$ and $33.3 \%$ respectively). ${ }^{2}$ Many characteristics have been shown to help in predicting the development of neovascularisation, such as the presence of a relative afferent pupillary defect, signs of significant retinal ischaemia on fluorescein angiography, ${ }^{6}$ and characteristic changes in electroretinographic recordings. ${ }^{5}$ We have shown in this study, by conventional statistical analysis, that the presence of pre-existing POAG may also predict the onset of new vessel formation and neovascular glaucoma. This association is not dependent on age or the coincidental presence of other pathologies predisposing to CRVO such as diabetes in POAG patients. Other studies have failed to find a similar association. ${ }^{2,11}$ However, criteria for defining pre-existing POAG in these studies have either not been stated or have included only patients with an established past ophthalmic history. This possibly explains why the occurrence of POAG in our study population (44\%) was comparatively high although still within the accepted range (5.7-66\%). ${ }^{12}$ It might have been expected that adequate IOP control amongst the POAG patients would have reduced this enhanced risk. This was not found to be the case, however, perhaps suggesting that this risk is not related to the level of IOP as such but to some other pathological process in the glaucomatous eye.

The results of laser treatment in our study population were generally poor. In particular, regression occurred in only $23 \%$ of NVG cases, which does not compare favourably with the report by Brooks and Gillies ${ }^{3}$ of neovascular regression in about $60 \%$ of NVG cases. Our records document that in individual cases as much treatment as technically possible had been given. However, treatment was often limited by media opacities and poor laser energy uptake by oedematous retina. We conclude that more laser therapy would not have been practical and thus not a solution to this poor responsiveness.

Of the variables studied, this poor responsiveness was significantly associated only with advancing patient age and POAG. Logistic regression showed that the association between POAG and poor response was still signifi- cant even when the influence of advanced patient age was taken into account. Although there was much variation in the total amount of treatment applied, no appreciable difference was seen on comparing the treatment regimens used in the POAG sub-group with the rest of the study population; therefore, it seems unlikely that this poorer response was due to different treatment regimens. An association between POAG and ocular hypoperfusion has been proposed ${ }^{13}$ which may enhance the ocular hypoxia produced in those who subsequently develop a CRVO. It is thought that the level of hypoxic drive determines not only whether new vessels will develop but also that higher levels lead to anterior rather than posterior segment neovascularisation. ${ }^{14}$ This possibly explains not only why CRVO patients with pre-existing POAG develop new vessels more frequently, but also why they tend to progress to neovascular glaucoma. Panretinal photocoagulation is thought to work by reducing hypoxic load, altering the balance of proliferative and inhibitory factors in favour of neovascular regression. ${ }^{14}$ In the presence of POAG the hypoxic drive may be beyond that which can be dealt with by laser therapy. There has been controversy in the literature regarding the effectiveness of panretinal photocoagulation at preventing or reversing ocular neovascularisation.,7 These studies have always grouped together all cases of neovascularisation after CRVO as the same pathological process. We have shown in this study that cases with pre-existing POAG are significantly less likely to respond to this treatment, suggesting a more aggressive pathology amongst this sub-group. This has not previously been taken into account and different proportions of POAG patients in the various studies may in part explain the conflicting reports on effectiveness.

It has been said that CRVO patients who develop neovascular complications, specifically NVG, often have blind, painful eyes which require retrobulbar alcohol or enucleation. ${ }^{15}$ This was not found to be the case in this study. Only 12 patients had acuities recorded as worse than 'hand movements' at 2 years; thus most retain some navigable vision. Also, only one eye required enucleation for pain. All other patients, including 28 NVG cases, remained comfortable with a combination of medical,

Table III. Visual acuities after 2 years in those with and without pre-existing POAG

\begin{tabular}{lccc}
\hline & $\begin{array}{c}\text { Worse than } \\
\text { 'hand movements' }\end{array}$ & $\begin{array}{c}\text { 'Hand movements' } \\
\text { to } 6 / 60\end{array}$ & Better than 6/60 \\
\hline POAG & 6 & 14 & 12 \\
Non-POAG & 8 & 15 & 18 \\
\hline
\end{tabular}


laser or surgical treatment, suggesting that prognosis in NVG secondary to CRVO is better than previously thought.

In conclusion, we confirm that ocular neovascularisation is a common sequela of CRVO and find that this complication responds poorly to panretinal photocoagulation. However, most of those affected retain comfortable eyes with some useful vision. In the presence of pre-existing POAG, a CRVO is significantly more likely to develop ocular neovascularisation and NVG, which in turn will be less likely to respond to conventional panretinal photocoagulation. This enhanced complication rate is not reduced by adequate prior treatment of IOP. Poor responsiveness to laser treatment is also related to advancing age.

The authors would like to thank Mrs. C. Owen for administrative work and the Department of Public Health, Liverpool University, for advice on statistical analysis.

Key words: Central retinal vein occlusion, Neovascularisation, Primary open angle glaucoma.

\section{REFERENCES}

1. Glaser B. Extracellular modulating factors and the control of intraocular neovascularisation. Arch Ophthalmol 1988; 106:603-7.

2. Hayreh SS, Rojas P, Podhajsky P, Montague P, Woolson RF. Ocular neovascularization and retinal vascular occlusion. III. Incidence of ocular neovascularization with retinal vein occlusion. Ophthalmology 1983;90:487-506.

3. Brooks AMV, Gillies WE. The development and management of neovascular glaucoma. Aust NZ J Ophthalmol 1990;18:179-85.
4. Magargal LE, Brown GC, Augsburger JJ, Parrish RK. Neovascular glaucoma following central retinal vein occlusion. Ophthalmology 1981;88:1095-1101.

5. Morrell AJ, Thompson DA, Gibson JM, Kritzinger EE, Drasdo N. Electroretinography as a prognostic indicator of neovascularisation in CRVO. Eye 1991;5:362-8.

6. Hayreh SS, Klugman MR, Beri M, Kimura AE, Podhajsky $P$. Differentiation of ischemic from non-ischemic central retinal vein occlusion during the early acute phase. Graefes Arch Clin Exp Ophthalmol 1990;228:201-17.

7. Hayreh SS, Klugman MR, Podhajsky P, Servais GE, Perkins ES. Argon laser panretinal photocoagulation in ischemic central retinal vein occlusion. A 10-year prospective study. Graefes Arch Clin Exp Ophthalmol 1990;228:281-96.

8. Hayreh SS, March W, Phelps CD. Ocular hypotony following retinal vein occlusion. Arch Ophthalmol 1978;96:827-33.

9. Kanski JJ. Clinical ophthalmology. London: Butterworth, 1987:7.7.

10. Altman DG. Practical statistics for medical research. London: Chapman and Hall, 1991.

11. Sinclair SH, Gragoudas ES. Prognosis for rubeosis iridis following central retinal vein occlusion. Br J Ophthalmol 1979;63:735-43.

12. Luntz MH, Schenker HI. Retinal vascular accidents in glaucoma and ocular hypertension. Surv Ophthalmol 1980;25:163-7.

13. O'Brien C, Saxton V, Crick RP, Meire ADN. Doppler carotid artery studies in asymmetric glaucoma. Eye 1992;6:273-6.

14. Murdoch IE, Rosen PH, Shilling JS. Neovascular response in ischaemic central retinal vein occlusion after panretinal photocoagulation. Br J Ophthalmol 1991;75:459-61.

15. Vernon SA. Panretinal cryotherapy in neovascular glaucoma. In: Mills KB, editor. Glaucoma. Oxford: Pergamon Press, 1989:215-9. 